Article

\title{
Genome-Wide Analysis of the Role of NAC Family in Flower Development and Abiotic Stress Responses in Cleistogenes songorica
}

\author{
Xifang Zong, Qi Yan, Fan Wu, Qian Ma and Jiyu Zhang *(1) \\ State Key Laboratory of Grassland Agro-Ecosystems, Key Laboratory of Grassland Livestock Industry \\ Innovation, Ministry of Agriculture and Rural Affairs, College of Pastoral Agriculture Science and Technology, \\ Lanzhou University, Lanzhou 730020, China; zongxf15@lzu.edu.cn (X.Z.); yanq16@lzu.edu.cn (Q.Y.); \\ wuf15@lzu.edu.cn (F.W.); maq18@lzu.edu.cn (Q.M.) \\ * Correspondence: zhangjy@lzu.edu.cn; Tel.: +86-138-9332-9958
}

Received: 22 July 2020; Accepted: 11 August 2020; Published: 12 August 2020

\begin{abstract}
Plant-specific NAC (NAM, ATAF, CUC) transcription factor (TF) family plays important roles in biological processes such as plant growth and response to stress. Nevertheless, no information is known about NAC TFs in Cleistogenes songorica, a prominent xerophyte desert grass in northwestern China. In this study, 162 NAC genes were found from the Cleistogenes songorica genome, among which 156 C. songorica NAC (CsNAC) genes (96.3\%) were mapped onto 20 chromosomes. The phylogenetic tree constructed by CsNAC and rice NAC TFs can be separated into 14 subfamilies. Syntenic and $\mathrm{Ka} / \mathrm{Ks}$ analyses showed that CsNACs were primarily expanded by genomewide replication events, and purifying selection was the primary force driving the evolution of CsNAC family genes. The CsNAC gene expression profiles showed that 36 CsNAC genes showed differential expression between cleistogamous (CL) and chasmogamous (CH) flowers. One hundred and two CsNAC genes showed differential expression under heat, cold, drought, salt and ABA treatment. Twenty-three CsNAC genes were commonly differentially expressed both under stress responses and during dimorphic floret development. Gene Ontology (GO) annotation, coexpression network and qRT-PCR tests revealed that these $C_{S} N A C$ genes may simultaneously regulate dimorphic floret development and the response to stress. Our results may help to characterize the NAC transcription factors in C. songorica and provide new insights into the functional research and application of the NAC family in crop improvement, especially in dimorphic floret plants.
\end{abstract}

Keywords: Cleistogenes songorica; NAC; genome-wide; abiotic stress; cleistogamous

\section{Introduction}

The agricultural yield is affected by environmental factors. Stress incidents pose huge challenges to global agricultural production, causing many losses every year [1]. Plants may suffer from various environmental stresses at different stages of growth [2]. To survive in these severe environments, plants evolve complex genetic mechanisms that regulate gene expression through accurate transcriptional control and precise signal transduction.

Transcription factors (TFs) combine with corresponding cis-acting elements to activate or inhibit the expression of their target genes and are crucial regulatory factors in many signaling networks [3]. TFs are involved in the regulation of many biological processes, including cellular morphogenesis, metabolic process, signal transduction and stress response [4]. The NAC (NAM, ATAF and CUC2) family is one of the largest families of plant-specific transcription factors and exists widely in numerous plants $[5,6]$. The NAC gene was firstly reported to be involved in forming shoot apical meristem and 
primordium in Petunia hybia [7]. Generally, the NAC family possesses a highly conserved N-terminal region (NAM domain), and there are at least five subdomains [5]. Furthermore, the relatively divergent C-terminal transcriptional regulation region of NAC TFs can repress or activate the transcription of multiple target genes [8]. In addition, some NAC proteins possess $\alpha$-helical transmembrane motifs (TMs) at their C-terminus for binding to the plasma membrane [9,10].

The NAC TFs are implicated in plant growth and development processes, including shoot apical meristem formation [7], flower morphogenesis [11], leaf senescence, hormonal signaling [12], stomatal closure [13], plant height [14], cell division and metabolism [15,16], secondary cell wall biosynthesis [17], lateral root formation [18], seed development and germination [19,20], fruit ripening [21] and crop yield and quality [22]. The leaf aging of transgenic Arabidopsis overexpressing the VND-INTERACTING2 (VNI2; an NAC transcription factor) gene was delayed, while leaf aging of VNI2-deficient mutants was significantly accelerated [23]. Inhibition of the expression of ZmNAC130 resulted in the reduction in starch in maize [20]. MdNAC1 was found to be associated with plant height, and overexpression of the gene resulted in a dwarf phenotype in transgenic apple plants. Furthermore, NAC TFs are involved in responding to multiple stresses, such as pathogen immunity [24], salt [25], drought [26], cold [27] and heat [28]. The membrane-bound protein NTL8 induced by salt stress can regulate Arabidopsis seed germination through the gibberellin acid (GA) pathway. Germination of T-DNA inserted ntl8-1 mutant seeds resistant to paclobutrazol (PAC; GA biosynthetic inhibitor) and high salinity [19]. The nucleus-localized protein ONAC066 can activate the expression of OsDREB2A (a drought-responsive gene) by combining with the AtJUB1 binding site in the OsDREB2A promoter. Transgenic rice lines overexpressing ONAC066 showed improved tolerance to oxidative and drought stress and increased abscisic acid (ABA) sensitivity [26]. In soybean, the overexpression of GmNAC20 enhances salt and freezing tolerance and accelerates the formation of Arabidopsis lateral roots, while the overexpression of GmNAC11 only improves salt tolerance [29]. TaNAC30, a transcriptional activator in the wheat nucleus, was induced in a compatible wheat-Pst interaction and negatively regulated Pst resistance in wheat by restraining the expression of pathogenesis-related genes and the accumulation of hydrogen peroxide $\left(\mathrm{H}_{2} \mathrm{O}_{2}\right)$ [30]. Although this family was studied in numerous species, there are still few research studies on the role of NAC TFs in plant flowering. In addition, we have not elucidated the molecular mechanism of NAC TFs to perform those functions in Cleistogenes songorica.

C. songorica is an important forage and ecological grass widely distributed in desert areas of Northwest China, with an annual rainfall between 100 and $200 \mathrm{~mm}$ [31]. C. songorica is known for its extreme stress tolerance and dimorphic floret [32,33]. Overexpression of the CsALDH gene improves salt and drought resistance in transgenic Arabidopsis thaliana and alfalfa [34,35]. There are two different types of flower of chasmogamous $(\mathrm{CH})$ and cleistogamous (CL) in different positions of $C$. songorica. There are many differences in the morphology of pollen between dimorphic florets, such as pollen germination aperture dimensions and exine ornamentation [36]. CL transgenic cultivars are a strategy to reduce environmental risks caused by transgenic plants. Clarifying the molecular mechanism of NAC transcription factors in response to abiotic stress and CL flowering in C. songorica could offer effective support for the genetic improvement of crops. However, notably, no NAC genes have been functionally characterized in C. songorica. In this research, we performed the genomewide identification, chromosome location, phylogenetic classification, gene structure, expression analysis and coexpression networks of the NAC TFs in C. songorica. Furthermore, CL flowering-related and abiotic stress-associated genes were also investigated.

\section{Materials and Methods}

\subsection{Identification of NAC Genes in C. songorica}

The genome sequences and RNA-seq data of C. songorica were obtained from Lanzhou University. The Arabidopsis thaliana and rice NAC gene sequences were obtained from the Phytozome database (https://phytozome.jgi.doe.gov/pz/portal.html) and were used to identify homologous peptides from the 
C. songorica genome by using the BLAST program. An online website (http://weizhongli-lab.org/cd-hit) was used to remove redundant genes with the default setting. NAC TFs which only contained the NAM domain (PF02365) were predicted using the Hidden Markov Model in the Pfam database.

\subsection{Chromosome Localization, Gene Duplication and Syntenic Analysis}

All CsNAC genes were labeled to $C$. songorica chromosomes using the Mapchart program (https://www.wur.nl/en/show/Mapchart.htm). OrthoMCL soft V5 was used to identify the duplicated genes with default setting. The syntenic relationships of CsNAC and OsNAC genes were illustrated with Tbtools (https://github.com/CJ-Chen/TBtools). The $\mathrm{Ka}, \mathrm{Ks}$ and $\mathrm{Ka} / \mathrm{Ks}$ values were calculated by the PAML yn00 NG model (http://abacus.gene.ucl.ac.uk/software/paml.html). Based on the rice $\lambda$ value $\left(6.5 \times 10^{-9}\right)$, the divergence time of $N A C$ duplicated genes was calculated $\left(\mathrm{T}=\mathrm{Ks} / 2 \lambda \times 10^{-6} \mathrm{Mya}\right)$.

\subsection{Protein Properties, Conserved Motif, Gene Structure and Phylogenetic Analysis}

The sequence length, molecular weight and isoelectric point of each NAC protein were analyzed by ExPasy (http://www.expasy.org/tools/). Orthologous genes in Arabidopsis thaliana of CsNAC genes were predicted using the BLASTP program at TAIR (https://www.arabidopsis.org). A neighbor-joining (NJ) phylogenetic tree was constructed using the MEGA7 program (1000 bootstrap replicates). The gene structure of CsNAC genes was visualized using the GSDS tool (http://gsds.cbi.pku.edu.cn/index.php). The MEME online website (http://meme.ebi.edu.au) was used to predict CsNAC gene conserved motifs.

\subsection{Gene Expression Analysis in Various Tissues and under Multiple Stress Treatments}

C. songorica was planted according to a previous report [37]. All seedlings were grown in a growing room at $26 \pm 2{ }^{\circ} \mathrm{C}$, under photosynthetically active radiation of $150 \mu \mathrm{mol} \mathrm{m} \mathrm{m}^{-2} \mathrm{~s}^{-1}$, a $16 \mathrm{~h}$ photoperiod and $65 \%$ relative humidity. Each pot contained $450 \mathrm{~g}$ of sand/vermiculite mixture, and the seedlings were watered with $100 \mathrm{~mL}$ Hoagland's solution every 3 days. Nine week-aged plants were treated with heat $\left(40{ }^{\circ} \mathrm{C}\right)$, cold $\left(4^{\circ} \mathrm{C}\right)$, low salt $(50 \mathrm{mM} \mathrm{NaCl})$, middle salt $(100 \mathrm{mM} \mathrm{NaCl})$, high salt $(200 \mathrm{mM} \mathrm{NaCl})$ and ABA (100 $\mu \mathrm{mM}$ ABA) for $24 \mathrm{~h}$. Eleven week-aged plants were treated with low drought (LD, 6-10\% soil water content) and high drought (HD, 1-3\% soil water content) for 2 weeks and recovery $48 \mathrm{~h}$ after high drought. Shoots and roots were collected after treatment, frozen immediately in liquid nitrogen and stored at $-80^{\circ} \mathrm{C}$. The expression levels (FPKM) of the CsNAC genes in various tissues (roots, leaves, seeds, cleistogamous (CL) flowers and chasmogamous $(\mathrm{CH})$ flowers) and multiple stress treatments were obtained from RNA-seq data (unpublished data). The ln-transformed values for the FPKM of CsNAC genes were used for expression profile analysis at Omicshare (http://www.omicshare.com).

Total RNA was extracted from $C$. songorica shoots under four treatments (high temperature, low temperature, salt, drought stress) using the Sangon Biotech RNAiso reagent kit. The extracted RNA was removed underlying genomic DNA and then reverse-transcribed into first-stand cDNA using the TaKaRa reagent Kit. PerlPrimer v1.1.21 software was used to design specific primers based on the CsNAC gene sequences. The qRT-PCR reaction was performed according to the instructions of the $2 \times$ TaqMan Fast qPCR Master Mix kit (Sangon Biotech, Shanghai, China). The correlative expression was calculated according to the $\Delta \triangle \mathrm{Ct}$ method. The expression quantity of CsGADPH gene was used as an internal control [38].

\subsection{Coexpression Network Construction and Gene Annotation Analysis}

The coexpression network of CsNAC genes was constructed using R package (WGCNA; weighted gene coexpression network analysis). Fifty-nine expression data sets were applied for the stress-related coexpression analysis, and 14 expression data sets were used for the CL flower development coexpression analysis. Coexpression networks were displayed by Cytoscape v 3.7.2. KOBAS 3.0 was used to annotate coexpressed genes. 


\section{Results and Discussion}

\subsection{Identification of NAC TFs in C. songorica}

C. songorica is an important native perennial forage and ecological grass in Northwest China. In recent years, we performed whole-genome sequencing of $C$. songorica and conducted a large number of transcriptome sequences to mine its genetic resources. In this research, we identified 162 NAC genes in C. songorica genome. The NAC genes were named CsNAC001 to CsNAC162 (Table S1). The number of NACs identified in C. songorica was similar to rice (151) [39], soybean (152) [40] and Vitis vinifera (163) [41] and redundant to Arabidopsis thaliana (117) [39] and Brachypodium distachyon (101) [42]. Overall, the results indicated that the NAC TFs did not undergo special expansion in C. songorica. Furthermore, the isoelectric points (pIs), amino acid residues and molecular weights (Mws) were analyzed. The 162 CsNAC proteins varied from 157 (CsNAC004) to 856 (CsNAC076) amino acid residues with an average of 378.5 aa. The relative Mws ranged from 17.8 (CsNAC004) to 96.1 (CsNAC076) $\mathrm{kDa}$ with an average of $41.8 \mathrm{kDa}$, and the pIs ranged from 4.48 (CsNAC003) to 11.3 (CsNAC063) with 101 members showing isoelectric point ( $\mathrm{pI}$ ) $\leq 7$ and the remainders showing pI $>7$ (Table S1), suggesting that NAC TFs may act under a different physiological environment [10].

\subsection{Phylogenetic Relationship and Evolutionary Analysis of CsNAC and OsNAC TFs}

To determine the evolutionary relationships between CsNAC and OsNAC proteins, a neighbor-joining phylogenetic tree was performed. As shown in Figure 1, CsNAC proteins were separated into two major groups-A and B (Figure 1). The result is consistent with a previous study [39]. Group A and B were separated into 7 subfamilies, respectively. Subfamily SNAC contained twenty CsNAC protein members, whereas only two CsNAC proteins were found in subfamily NEO (Figure 1). Each subfamily contains OsNAC and CsNAC proteins (Figure 1), which may indicate that no subfamily loss occurred in the NAC family after $C$. songorica and rice differentiation. The subfamily SNAC is related to the plant response to stress [43], and contains 13 OsNAC proteins and 20 CsNAC proteins (Figure 1), which indicates that the NAC gene from C. songorica is more closely related to stress than rice. Most subfamilies contain paralogous gene pairs, such as SNAC with 7 pairs, followed by subfamily ONAC4 with 6, but no paralogous gene pairs were found in NEO and ONAC1, and all of the paralogous gene pairs were from the same subfamily (Figures 1 and 2).

Syntentic analysis was usually used to identify homologous genes and evolutionary relationships between genes [44]. To explore the evolutionary relationships between rice and C. songorica NAC family, we performed a comparative syntenic analysis and found 68 OsNACs and 93 CsNACs as orthologs (Figure 2). All orthologous gene pairs come from the same subfamily (Figure 1), which provides potential support for the accuracy of the phylogenetic tree. Among orthologous genes, twenty-eight pairs of orthologous genes were one-to-one, including CsNAC013 - LOC_Os06g01230.1 and CsNAC156 LOC_Os12g43530.1. The results indicated these NACs evolved from the common ancestor of rice and C. songorica. We further analyzed the evolutionary pressure between orthologous genes (Cs-Os). The Ks values peaked at $0.6-1.8$ between $C$. songorica and rice (Figure $3 \mathrm{a}$ ). The $\mathrm{Ka} / \mathrm{Ks}$ ratios were primarily distributed at 0-0.36, with the maximum value being observed in the CsNAC059 - LOC_Os03g59730.1 pair $(\mathrm{Ka} / \mathrm{Ks}=0.62)$ (Figure 3b: Table S2), indicating that they undergo purifying selection during biological evolution between two species [37]. 


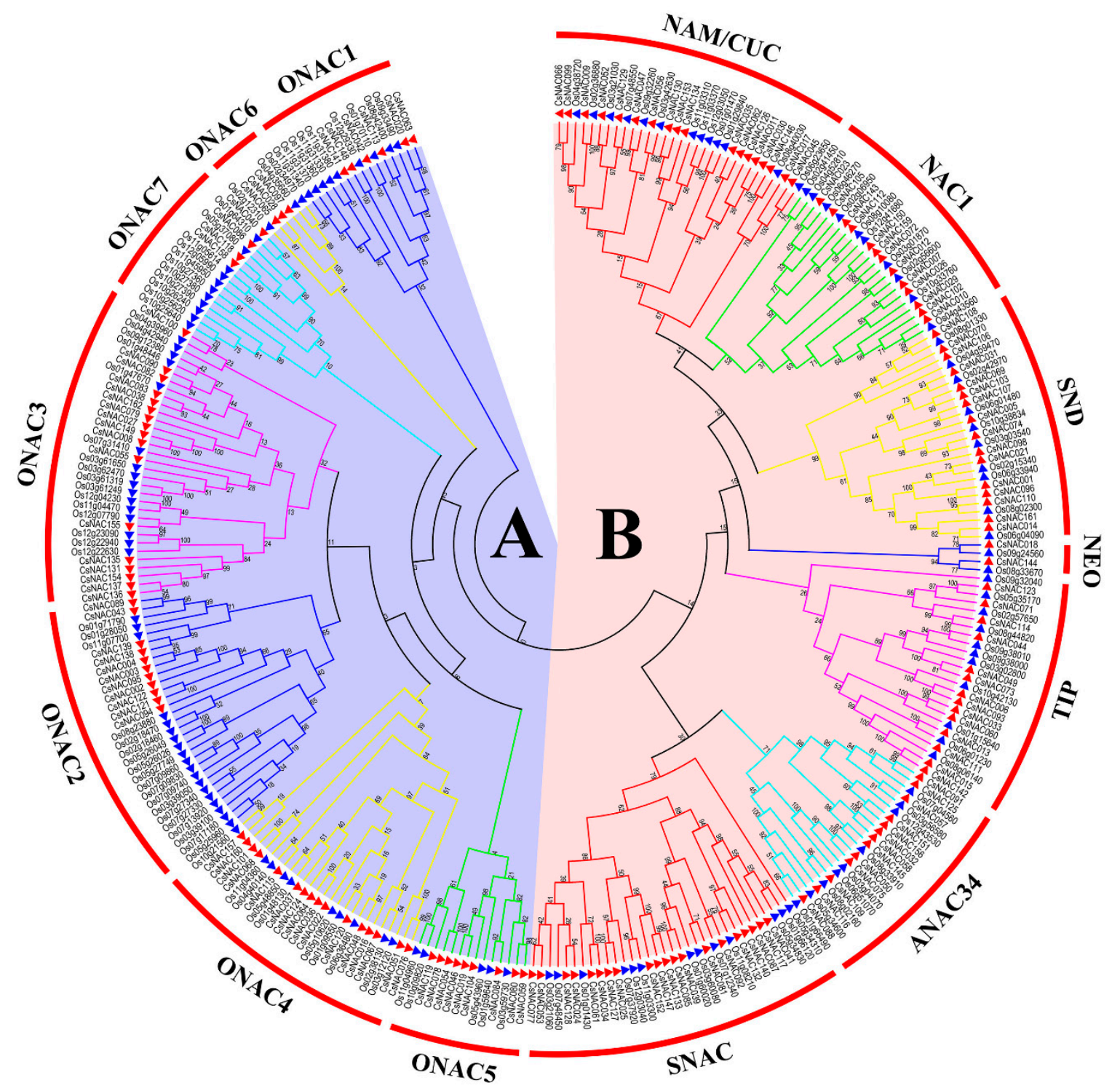

Figure 1. Phylogenetic tree of NAC transcription factors (TFs) in C. songorica and rice. The neighbor-joining (NJ) phylogenetic tree was constructed using the MEGA7 program with 1000 bootstrap replicates. CsNAC and OsNAC proteins were labeled by triangles with red and blue, respectively. 


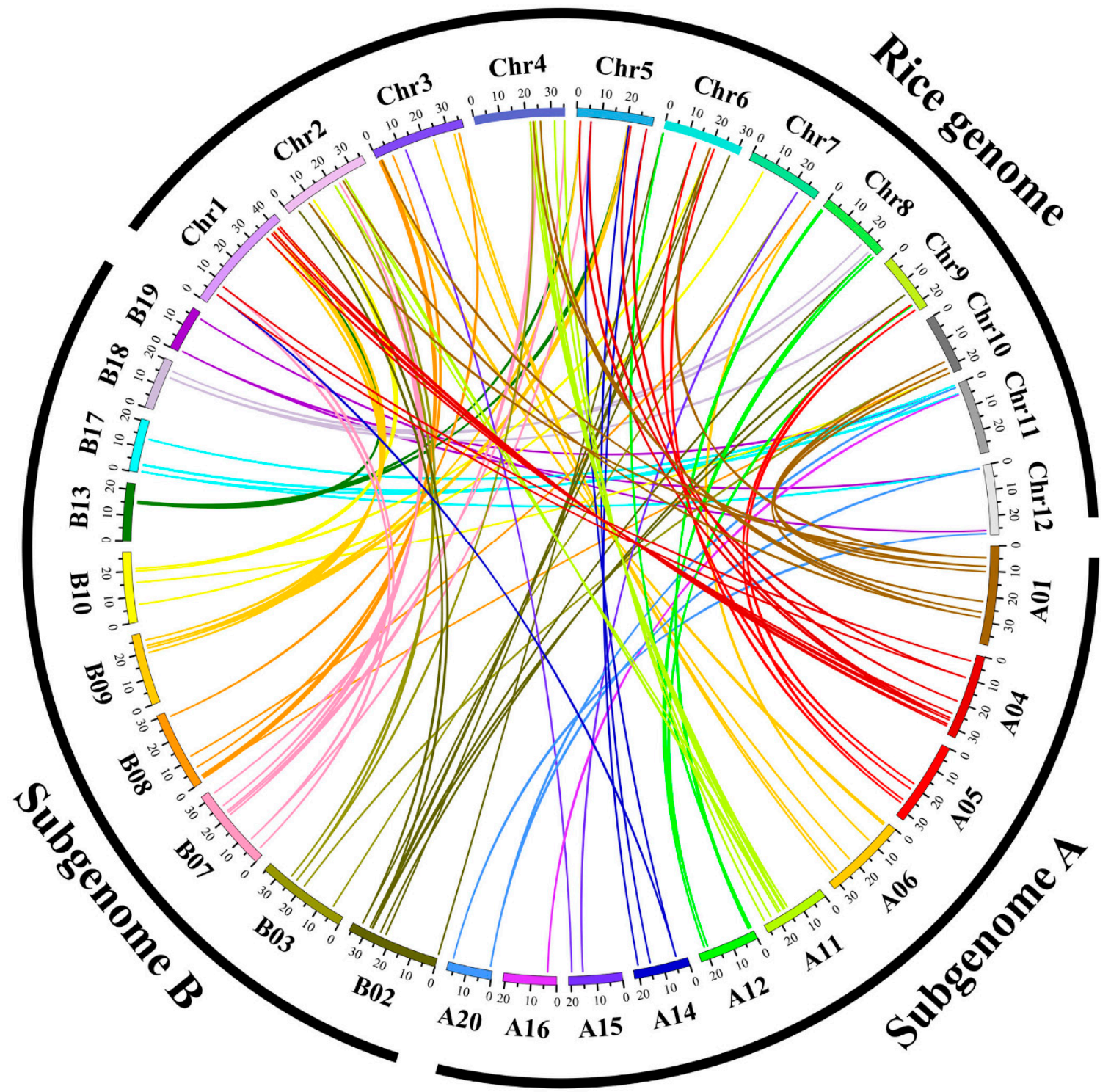

Figure 2. Schematic representations of syntentic relationships of $N A C$ members between $C$. songorica and rice. Colored lines indicate NAC orthologous genes in the C. songorica and rice genomes. Subgenomes $\mathrm{A}$ and B represent $20 \mathrm{C}$. songorica chromosomes, while Chr1 to Chr12 represent rice. The chromosome number is marked below each chromosome. 


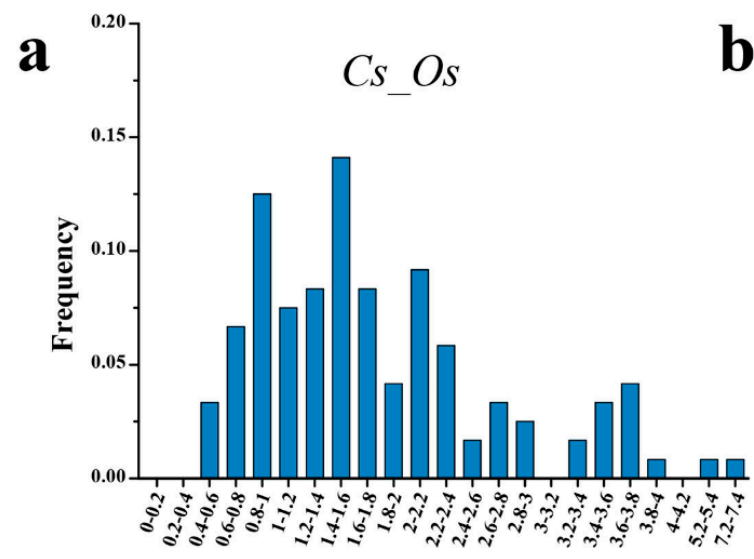

Range of Ks value

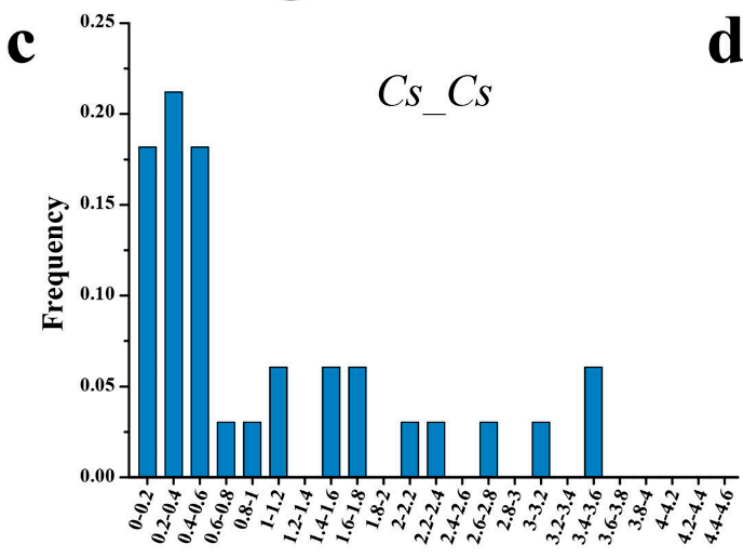

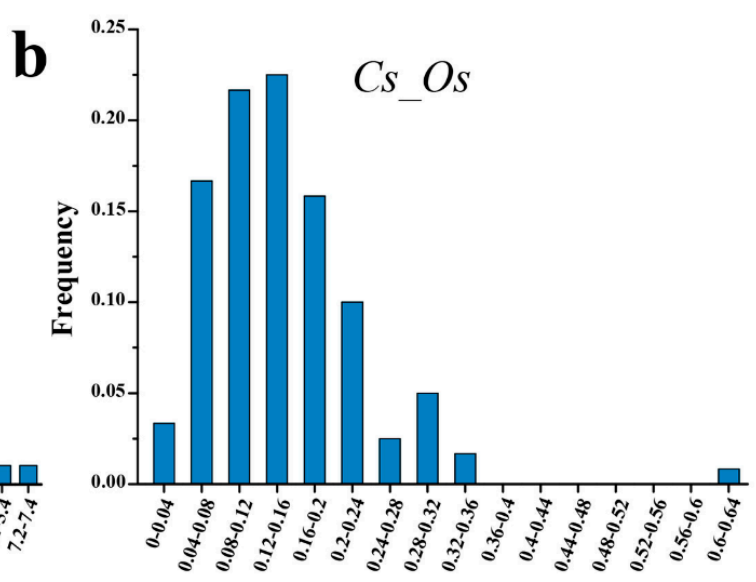

Range of $\mathrm{Ka} / \mathrm{Ks}$ value

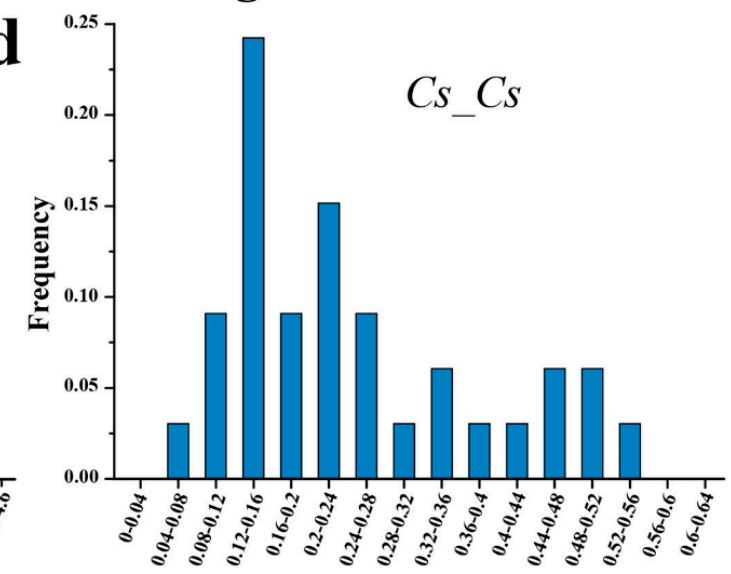

Figure 3. $\mathrm{Ks}$ and $\mathrm{Ka} / \mathrm{Ks}$ value distributions of the NAC members in C. songorica and rice genomes. The distribution of $\mathrm{Ks}$ and $\mathrm{Ka} / \mathrm{Ks}$ values of orthologous genes between $\mathrm{C}$. songorica and rice $(\mathbf{a}, \mathbf{b})$ and paralogous genes in C. songorica $(\mathbf{c}, \mathbf{d})$.

\subsection{CsNAC Gene Structures and Conserved Motifs}

A phylogenetic tree was performed with CsNAC protein sequences, which clustered the NAC proteins into 9 subgroups (Figure 4a). Gene structural diversity is a possible mechanism for the evolution of gene families [45]. To explore the structural diversity of CsNACs, we compared the intron/exon structure of each NAC gene in C. songorica. Generally, CsNAC genes with high bootstrap supported in the same subgroups that shared similar intron/exon structures in points of exon number and length (Figure $4 \mathrm{~b}$ ). For example, the CsNAC genes in subgroups III, V and VIII included 2 to 4 exons and those in subgroup II contained 3 to 5 exons. Inversely, subgroups VII and IX showed a large difference with exon numbers ranging from 1-14 (Figure 4a,b). These results indicated that the II, III, V and VIII subgroups are more conserved, while VII and IX could be related to the evolution of the CsNAC family.

To explore intron gain or loss information about the NAC family in C. songorica, we further performed the intron/exon structure of CsNAC paralogous gene pairs. Among 33 paralogous pairs, 25 pairs displayed similar intron/exon structure in points of either intron numbers or gene length (Figures $4 \mathrm{~b}$ and 5). Sixteen paralogous pairs displayed a highly conserved gene structure with the same exon numbers (Figure $4 \mathrm{~b}$ ). However, others showed a certain degree of difference. Nine CsNAC genes of the 33 paralogous pairs possessed one more exons than and a similar length to their paralogous genes (Figures $4 b$ and 5 ). We speculate that the differences may be due to single intron loss or gain that occurred during the structural evolution of NAC paralogs. 
a

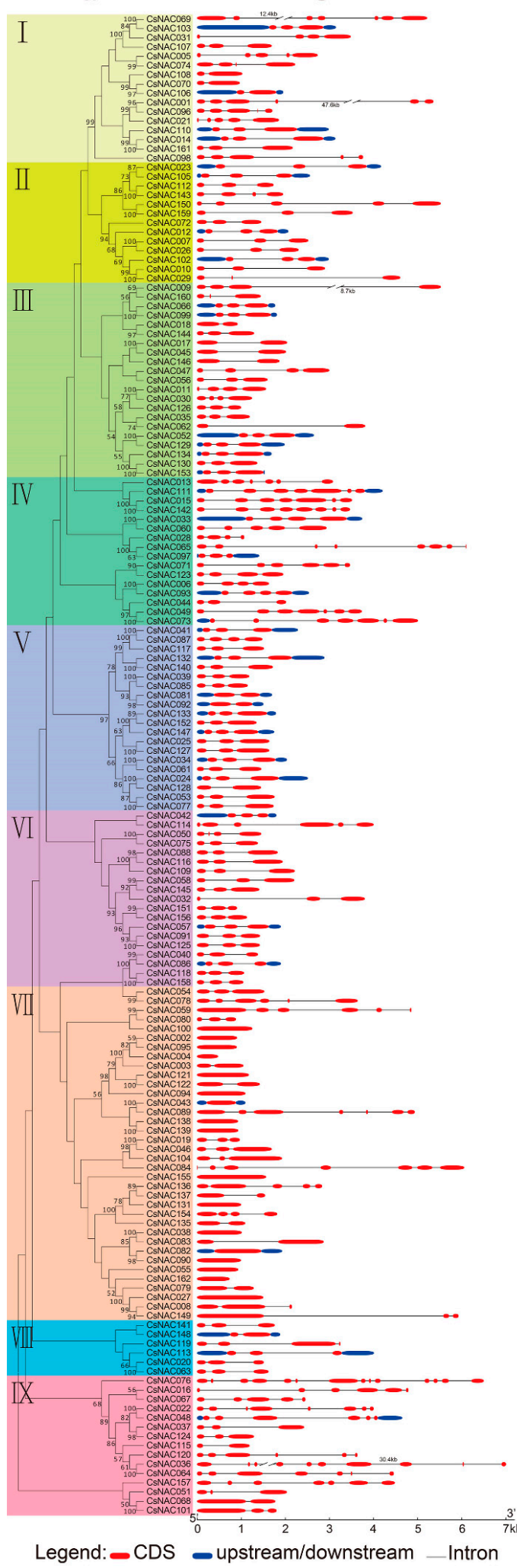

b

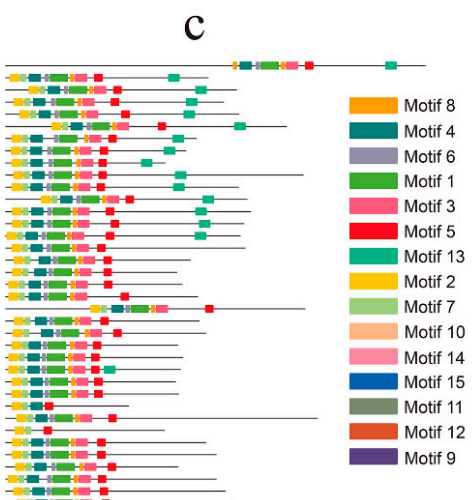

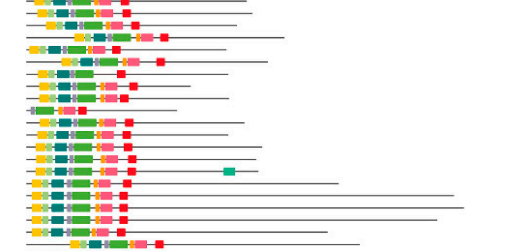
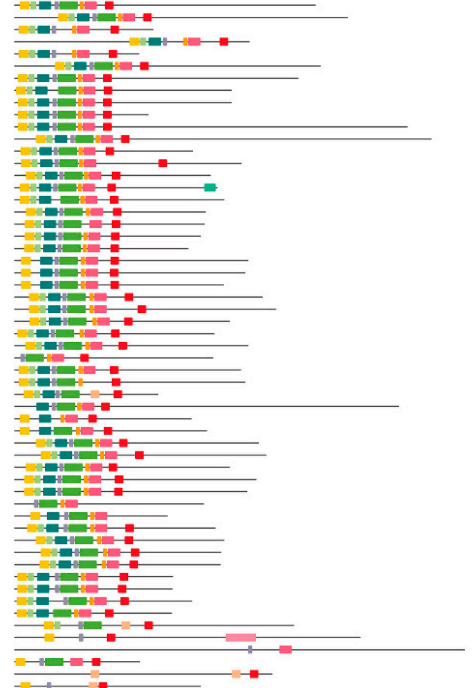

$=$

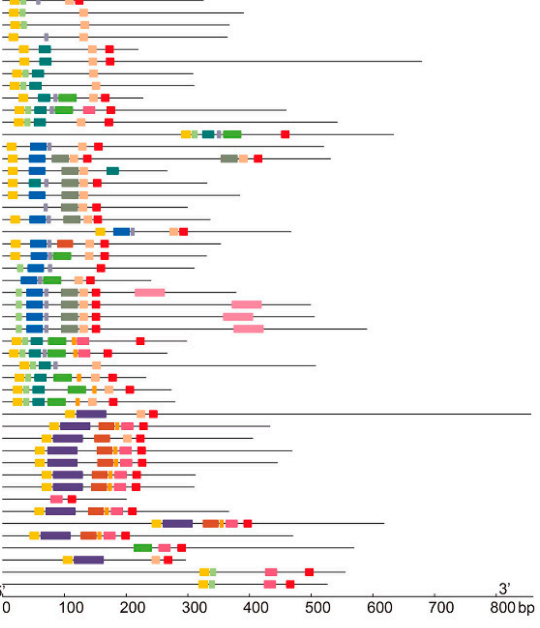

Figure 4. Phylogenetic relationships, gene structure and putative conserved domain distributions of the CsNAC family. (a) The phylogenetic tree was constructed with CsNAC protein sequences using MEGA 7 software. The cluster details are indicated in multiple colors. (b) Exon-intron structure is indicated by red rectangles and black lines, respectively. (c) Multiple colored boxes represent different motifs. The motif sequence information is provided in Figure S1. 


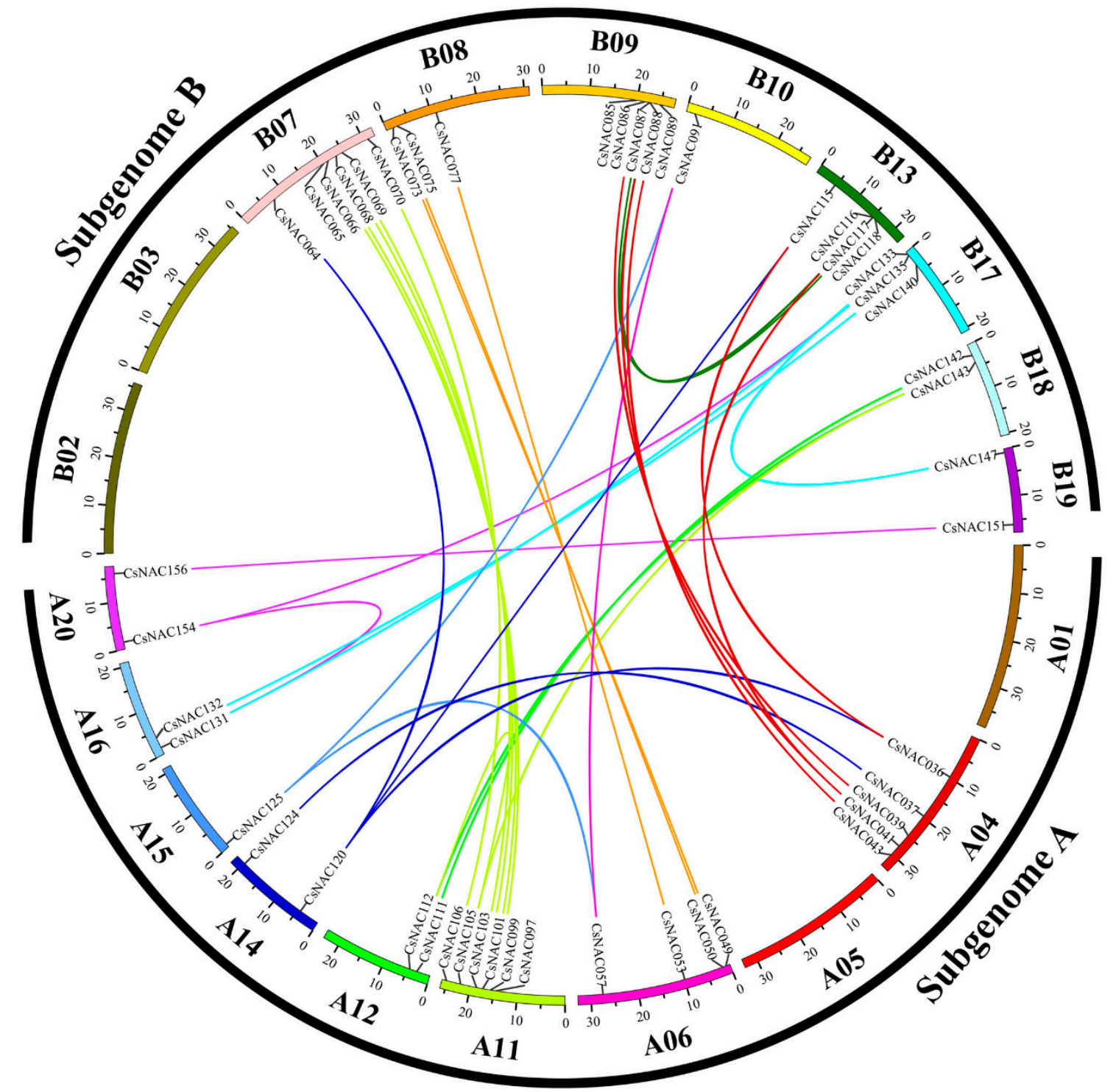

Figure 5. Distribution of the NAC duplicated genes in C. songorica. The colored lines within the chromosomes represent the syntentic relationships in C. songorica NAC members.

To further reveal the functional diversification of NAC transcription factors in C. songorica, conserved motifs were analyzed using the MEME program, and 15 conserved motifs were predicted. As expected, members with high bootstrap support in the phylogenetic tree possessed highly similar motif compositions (Figure 4c). Motifs 2, 4, 3, and 5, respectively, represent the A, B, D and E subdomains of the NAM domain, while 6 and 1 represent subdomain C. Subdomain A is related to the formation of functional dimers. The highly conserved positively charged subdomains $\mathrm{C}$ and $\mathrm{D}$ bind to DNA, while subdomains $\mathrm{B}$ and $\mathrm{E}$ are involved in the functional diversity of the NAC TFs [8]. In fact, NAC TFs can regulate the expression level of downstream genes through complex interactions between the DB domain, NARD and activation domain [46]. Some CsNAC proteins (such as CsNAC043, CsNAC037, CsNAC101) have only partial motif composition of the NAM domain, and we call this NAC-like protein (Figure 4c) [43]. Furthermore, motifs 11, 14 and 15 were unique to subgroup VII, motifs 9 and 12 were only found in subgroup IX, motif 13 was present mainly in subgroup I, and motif 10 was mainly in subgroups VII, VIII, IX (Figure 4a,c). The specific structure of these subgroups may confer their potential specific functions [47]. A total of $66.7 \%(22 / 33)$ NAC paralogous gene pairs possessed the same motif compositions as one another, indicative of functional 
similarities. Inversely, special motifs were observed for the remaining 11 pairs of NACs; for example, CsNAC053 and CsNAC077 are different in motif 3 (Figures 4c and 5).

\subsection{Genomic Locations and Duplication of the NAC Genes in C. songorica}

As shown in Figure 6, a total of 156 (96.3\%) CsNACs were unequally distributed on 20 chromosomes of $C$. songorica. Chromosomes A01 and B07 had the most CsNAC genes (21, 25.93\%), whereas there were only 3 genes (1.9\%) in chromosome A16 (Figure 4). Subgenomes A and B possessed 75 (42.3\%) and $81(50 \%)$ CSNAC genes, respectively (Figure 6).
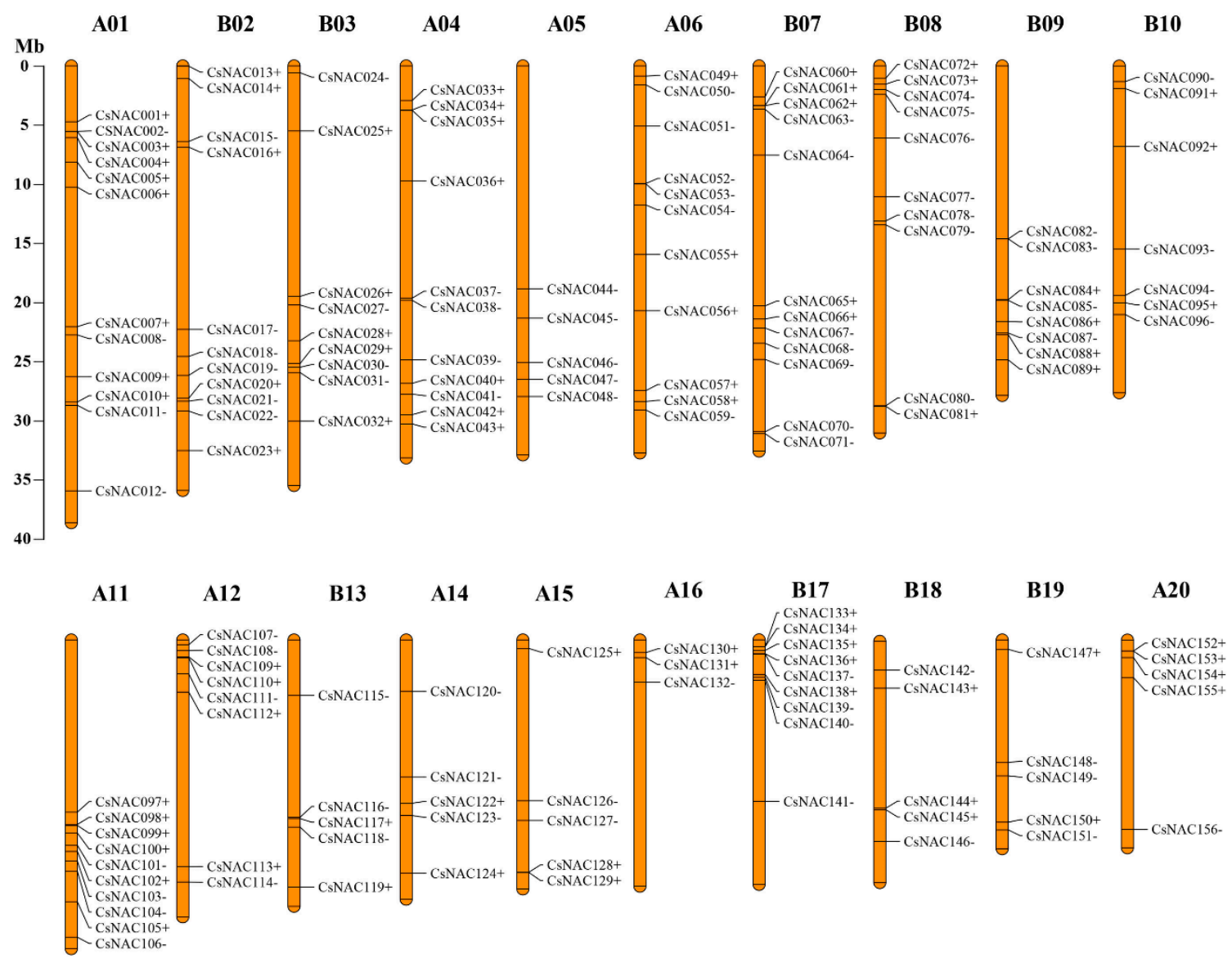

Figure 6. Chromosomal location of $C$. songorica NAC genes. The chromosome number is above each chromosome. The scale represents chromosome length.

Gene duplication is a mechanism for gene family expansion and new gene generation. In rice, gene family expansion is primarily involved in segmental duplication, tandem duplication, and transposition events [48]. To find NAC duplicated genes in C. songorica genome, the syntentic analysis was carried out using OrthoMCL software. In this research, 33 pairs of duplicated genes were found in 162 CsNAC genes (Figure 5). These duplicated genes were the most common on chromosomes B07 and A11, whereas there were no duplicated gene pairs on chromosomes A01, B02, B03 and A05 (Figure 5). All CsNAC paralogous gene pairs existed on different chromosomes, and no tandem duplication genes were found. A total of $72.7 \%$ CsNAC duplicated gene was found between subgenome A and B in C. songorica, which was the same as the bZIP family (72.5\%). In addition, a previous study indicated that $C$. songorica experienced a whole-genome duplication event during biological evolution [37]. These results suggested that whole-genome duplication events resulted in the expansion of the NAC family in C. songorica. NAC genes in pear undergo purification selection and positive selection while only undergoing purification selection in cotton $[49,50]$. In this study, we analyzed the evolutionary 
pressure between CsNAC duplicated genes (Cs-Cs) and found that the $\mathrm{Ka} / \mathrm{Ks}$ ratios of all $N A C$ duplicated genes were less than 1, indicating that NAC genes underwent purifying selection in the C. songorica genome (Figure 3d; Table S3). In addition, the frequency distributions of Ks value peaked at 0-0.6, and their divergence times were 2.0-272.4 MYA (Figure 3c; Table S3).

\subsection{Expression Profiles and Coexpression Network Analysis of CL Flowering Related CsNAC Genes}

We performed the expression profiles of CsNAC genes in multiple organs, including the seeds, leaves, roots, chasmogamous $(\mathrm{CH})$ and cleistogamous (CL) flowers (Figure 7a). A total of $112(69.1 \%)$ CsNAC genes had expression in at least one organ (FPKM $\geq 1)$, and $44(27.2 \%)$ CsNACs had expression in all organs (FPKM $\geq 1)$. Furthermore, $37(22.8 \%)$ CsNACs highly expressed in at least one organ (FPKM $\geq 20$ ) (Figure 7a; Table S4). Five CsNACs had high expression in all organs, including CsNAC041, CsNAC081, CsNAC087 CsNAC123 and CsNAC148 (Figure 7b; Table S4). Strikingly, 12 CsNACs had high expression in dimorphic florets but not in roots and leaves; 8 CsNACs had high expression in roots but not in dimorphic florets (Figure 7a; Table S4).

There are approximately 700 cleistogamy plants in the world, such as Pisum sativum and Impatiens balsamina. Cleistogamy was separated into three types: complete cleistogamy, dimorphic cleistogamy and induced cleistogamy [51]. Compared with chasmogamous, cleistogamy can produce seeds by self-pollination to maintain the population's reproduction under extreme environments. C. songorica is a dimorphic cleistogamy plant that produces open (chasmogamous) flowers at the apical meristem and closed (cleistogamous) flowers in the sheath. CL in the sheath has a significant impact on the seed yield of $C$. songorica [36]. Thirty-six CsNAC genes had differential expression $\mid \log _{2}$ (fold change) $\geq 1$ | between dimorphic florets, including 23 upregulated and 13 downregulated CsNACs (Figure 7c; Table S4). GO annotation indicated that these CsNACs were related to the biosynthetic process, response to stimulus, organ development, floral whorl development, anther development and dehiscence (Table S5). The NAM subfamily is involved in plant development. In this subfamily, EPHEM-ERAL1 (EPH1), positively regulates PCD (programmed cell death) during petal senescence in Japanese morning glory (Ipomoea) [52]. The sly-miR164-regulated SINAM2 genes play key roles in tomato floral-boundary specification [53]. The CsNAC011, CsNAC099, CsNAC130, CsNAC134 and CsNAC146 genes in the NAM subfamily showed differential expression between CL and CH flowers, and we speculate that they may also play vital roles in the cleistogamy of $C$. songorica.

Genes with similar expression patterns may be coregulated, functionally related, or in the same pathway. WGCNA can predict the regulatory relationship between genes and the function of unknown genes based on expression data, providing important clues for the study of unknown genes [54]. Nine dimorphic floret-related CsNAC genes (CsNAC011, CsNAC014, CsNAC025, CsNAC028, CsNAC061, CsNAC097, CsNAC105, CsNAC127 and CsNAC128) were used for coexpression analysis using WGCNA. A total of 2372 coexpressed genes were identified, and 917 coexpressed genes showed overlap with these 9 genes (Figure 7d; Table S6). GO annotation indicated that these CsNACs were related to the developmental process, reproductive process, flower development, pollen exine formation and response to stress (Table S6). The result indicated these CsNACs can be involved in dimorphic floret differentiation by regulating flower development and reproductive processes. 
$\mathbf{a}$

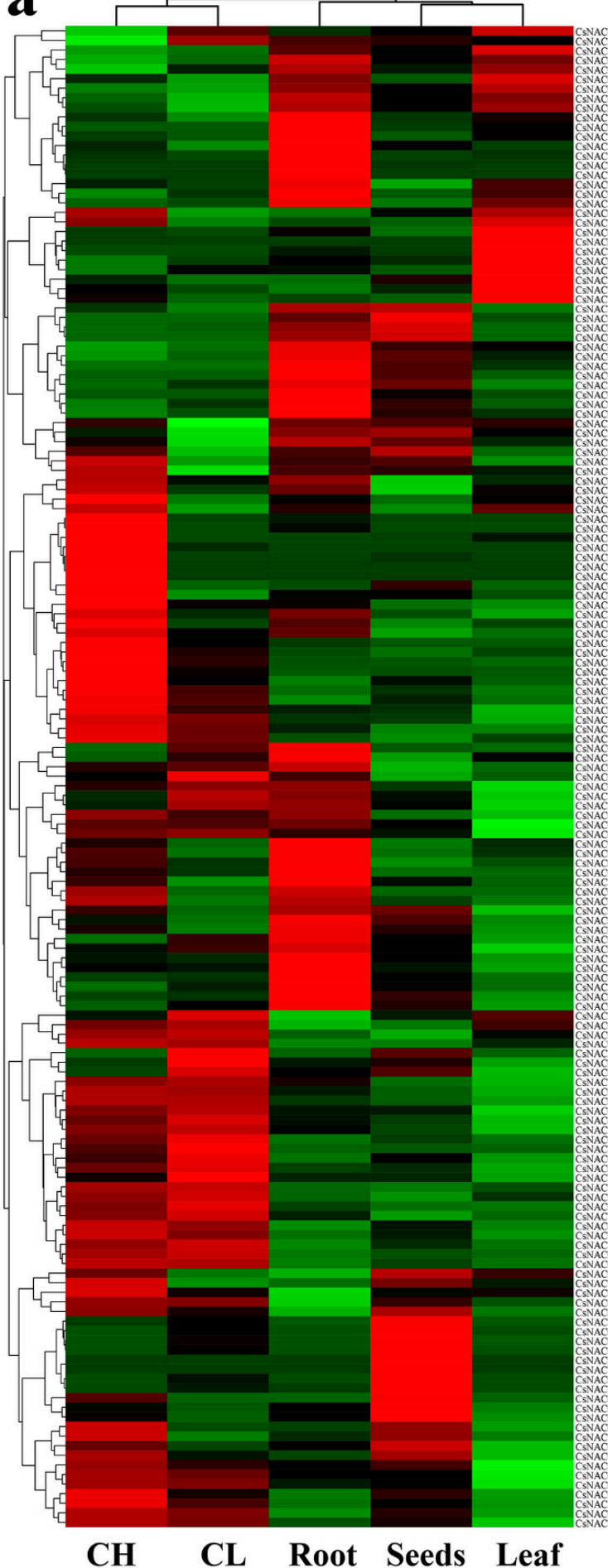

b

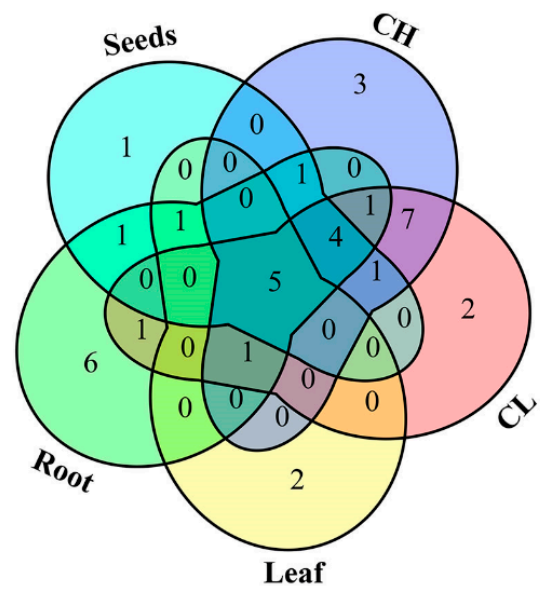

c
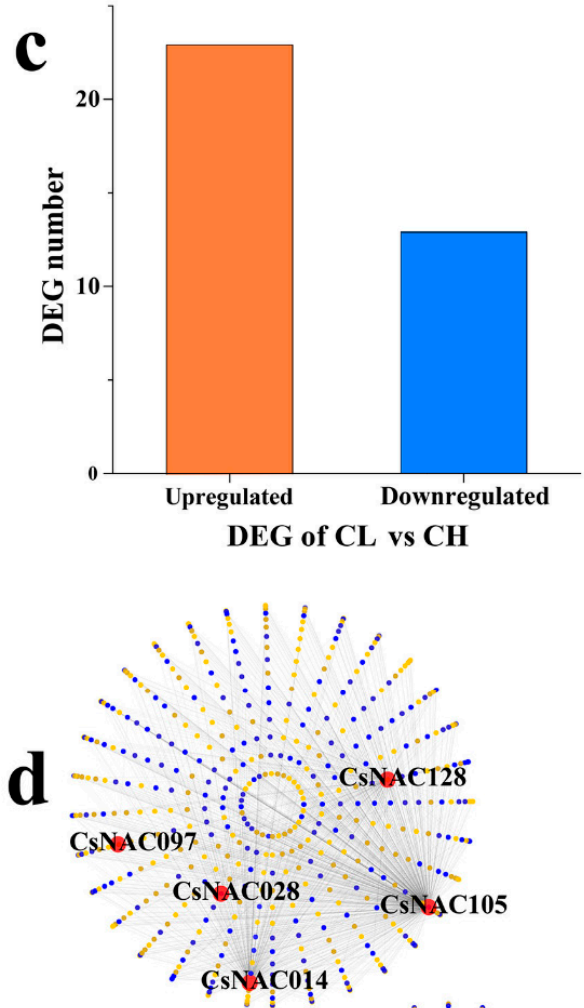

Figure 7. Expression profiles and coexpression network of CsNAC TFs during dimorphic floret development. (a) Hierarchical clustering of CsNACs in multiple organs based on transcriptome data. (b) The highly expressed CsNACs (FPKM $\geq 20$ ) in multiple organs was marked by the overlapping petal circle. (c) The number of up-regulated and down-regulated CSNAC differentially expressed genes (DEGs) in dimorphic florets development. (d) Coexpression network of CsNAC DEGs between cleistogamous $(\mathrm{CL})$ and chasmogamous $(\mathrm{CH})$ flowers. The light yellow points represent individual genes. The blue points represent overlapping genes. The dark yellow points represent yellow points overlying blue points. 


\subsection{Expression Profiles and Coexpression Network Analysis of Abiotic Stress-Related CsNAC Genes}

NAC transcription factors participate in plant response to various abiotic stresses. Overexpression of SNAC1 enhances transgenic rice drought resistance in the field during the reproductive stage [55]. The transgenic alfalfa overexpressing MfNAC3 had better cold resistance, and the expression levels of cold response genes $M t C B F s$ and $M t C A S$ s were significantly increased [27]. Although many stress-related NAC TFs have been studied in various species, there is still no research on $C$. songorica.

To explore the role of CsNAC genes in abiotic stress, we performed the expression profile of CsNAC genes in multiple abiotic stress-related treatments, including drought, high temperature, low temperature, salt and ABA. Eighty-one (50.0\%) and 96 (59.3\%) CsNACs had expression under at least one treatment $(\mathrm{FPKM} \geq 1)$ in shoots and roots (Figure 8a; Table S7), respectively. Moreover, 77 (47.5\%) and 93 (57.4\%) CsNACs showed differential expression $\mid \log _{2}$ (fold change) $\geq 1 \mid$ under at least one treatment in shoots and roots, respectively (Figure $8 \mathrm{~b}$ ).

A total of 59,40,16, 50 and 38 CsNACs had differential expression under high temperature, low temperature, salt, drought stress and ABA treatments in shoots, respectively. Sixty-eight, 47, 45, 57 and 29 CsNACs had differential expression under the heat, cold, salt, drought stress and ABA treatments in roots, respectively (Figure 8b). Interestingly, 7 and 6 of DEGs (differentially expressed genes) overlapped under five treatments in shoots and roots, respectively (Figure 8b). In total, 102 CsNACs had differential expression under five treatments in two organs (Figure 8d; Table S7). The results showed that CsNAC genes participated in regulating abiotic stress. A Venn diagram showed that more CsNACs responded to drought and high temperature than responded to low temperature and salt stress (Figure 8b), indicating that CSNAC genes might play crucial roles in the response to drought and high temperature in $C$. songorica. These finding may be observed because $C$. songorica usually grows in desert areas. The stress-related CsNACs were primarily distributed in subfamilies SNAC, ONAC4, ONAC3, NAC2 and ONAC1. Eighty percent of SNAC genes showed differential expression in shoots and roots under five treatments (Figure 1; Table S7), showing that the SNAC subfamily is closely related to abiotic stress. The result was consistent with the findings of a previous study [43]. We further analyzed the relationship between ABA-responsive and abiotic stress-responsive CsNAC genes. Fifty-six CsNACs responded to ABA and abiotic stress simultaneously, while 46 CsNACs specifically responded to abiotic stress (Figure $8 \mathrm{~d}$ ). The result indicated that more ABA-dependent CsNAC genes participated in the response to abiotic stress than $\mathrm{ABA}$-independent.

Ten stress-related CsNAC genes were used for the coexpression network analysis. There were 1582 coexpression genes in the coexpression network, of which 545 were overlapping coexpression genes (Figure 8c; Table S8). Moreover, we performed the GO annotation of 1582 coexpression genes, and found that some of these genes were related to stress-responsive GO terms, for example: the response to stress, response to hormone, defense response and oxidation-reduction process (Table S8). Strikingly, some coexpression genes were related to flower development, including reproductive process, flower development and photoperiodism flowering (Table S8).

We ulteriorly found that 23 CsNAC genes had differential expression in dimorphic florets and under abiotic stress (Figure 8e). Sang-Gyu Kim (2007) found that NTL8, an NAC transcription factor, regulates salt-responsive flowering via FLOWERING LOCUS T in Arabidopsis [56]. Furthermore, a previous study revealed that DEGs between CH and CL flowers were related to defense responses [57]. The results suggested that CL flowers could be induced by environment, while NAC transcription factors may be involved in related regulatory processes.

The expression patterns of some stress-related CsNAC genes were verified by qRT-PCR under drought ( $2 \%$ soil water content), salt (100 mM NaCl; $24 \mathrm{~h})$, high temperature $\left(40{ }^{\circ} \mathrm{C} ; 24 \mathrm{~h}\right)$ and low temperature $\left(4^{\circ} \mathrm{C} ; 24 \mathrm{~h}\right)$. The qRT-PCR results were in keeping with the transcriptome data Figure 9 indicating that these $8 \mathrm{CsNACs}$ were involved in four abiotic stresses. For example, CsNAC077, CsNAC112 and CsNAC143 were downregulated under drought and salt stress. CsNAC053 and CsNAC067 were upregulated under heat and cold stress. 


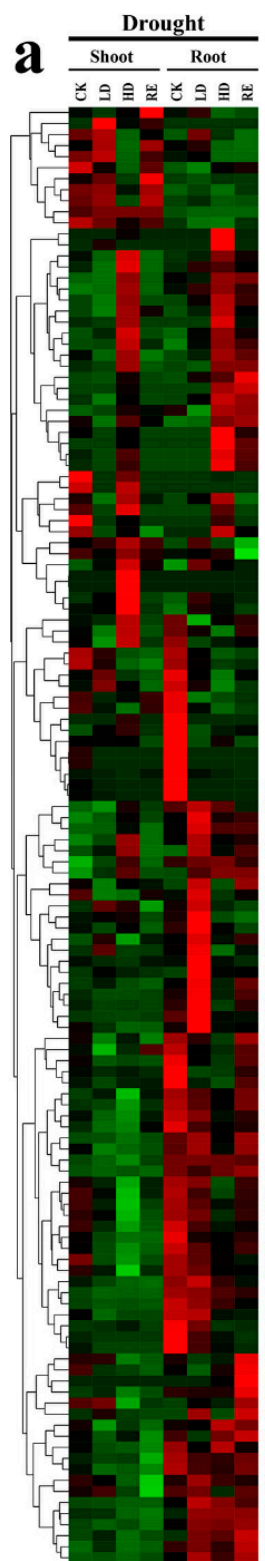

Temperature
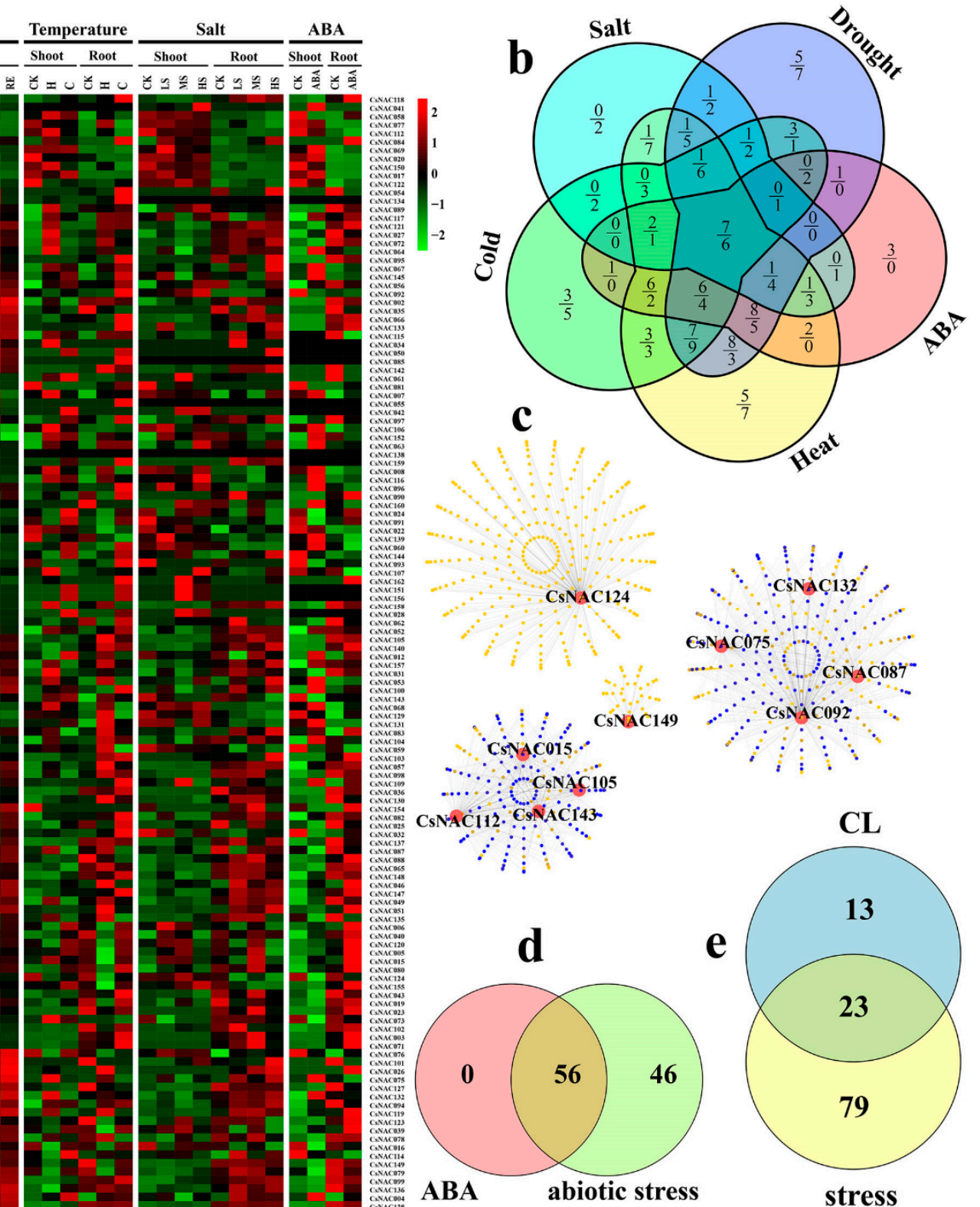

CsNACI12 ESNACITH
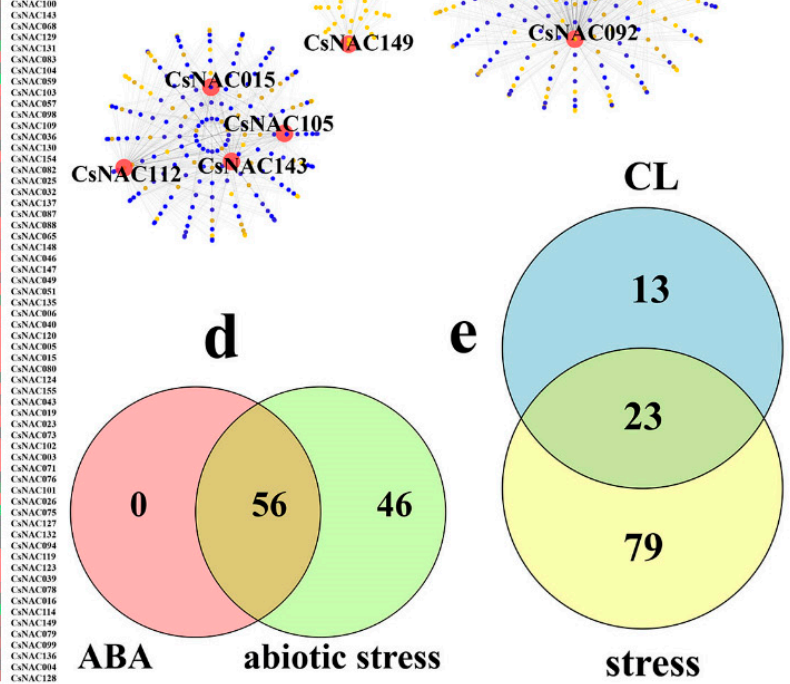

Figure 8. Expression profiles and coexpression network analysis of abiotic stress-associated CsNAC genes. (a) The expression profiles of CsNAC genes under five treatments (drought, heat, cold, salt and ABA). (b) The CsNAC DEGs under five treatments (high drought, heat, cold, high salt and ABA) were marked by the overlapping petal circle. The numbers above and below the line represent the CsNAC DEGs in shoots and roots, respectively. (c) Coexpression network of CsNAC genes with DEGs under abiotic stress. The light yellow points represent individual genes. The blue points represent overlapping genes. The dark yellow points represent yellow points overlying blue points. (d) The CsNAC DEGs under abiotic stress and ABA treatment was marked by the overlapping circle. (e) The CsNAC abiotic stress-associated and CL flowering-related genes were marked by the overlapping circle. CK: control; LD: low drought; HD: high drought; RE: recovery H: heat; C: cold; LS: low salt; MS: middle salt; HS: high salt. 

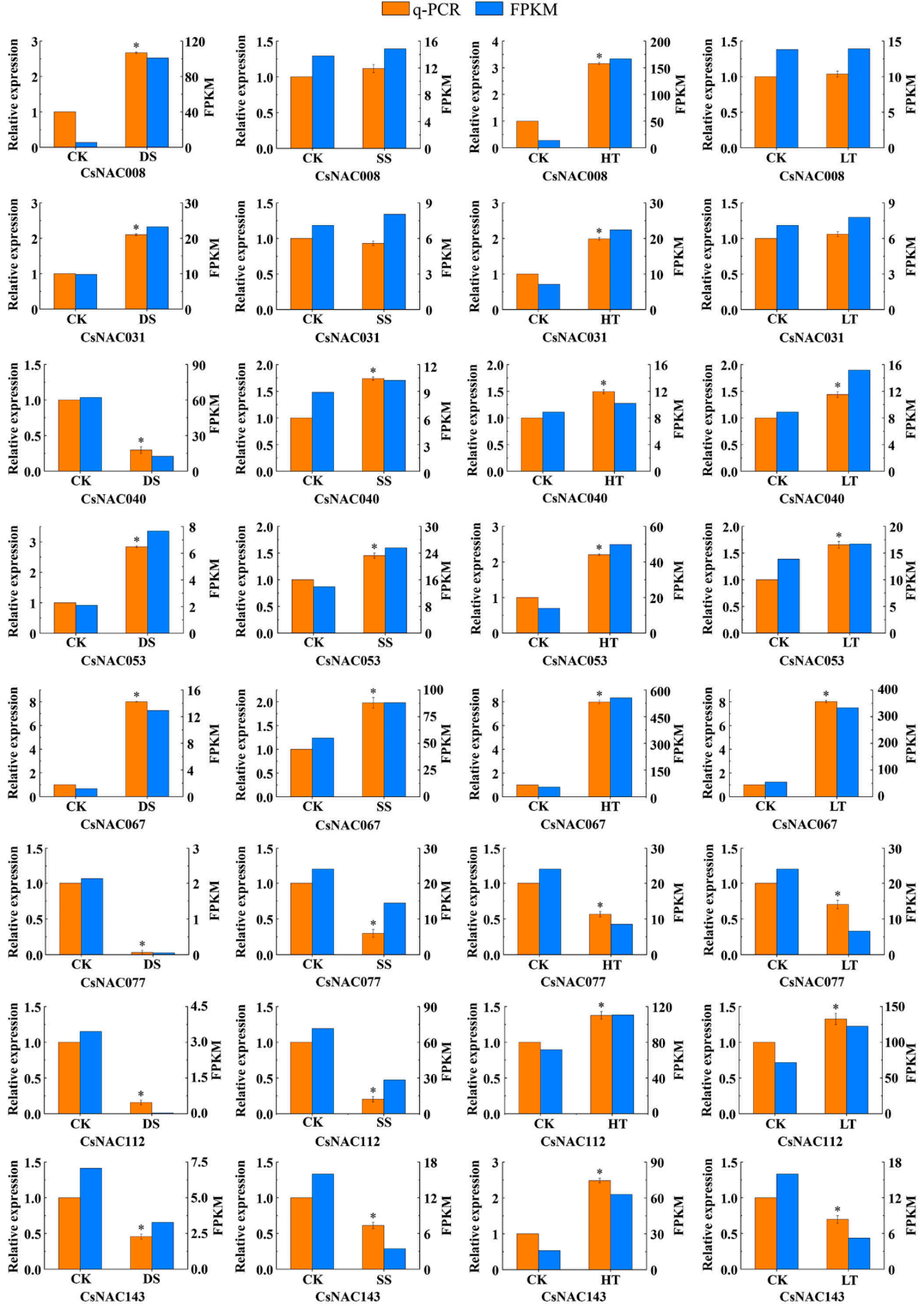

Figure 9. The expression of selected $C S N A C$ genes under abiotic stress in shoots by qRT-PCR. DS: drought stress; SS: salt stress; HT: high temperature; LT: low temperature. Bars represent means \pm standard error $(n=3)$. Asterisks indicate relative expression significant differences under abiotic stress $(p<0.05)$. The CsGAPDH was used as the internal control. 


\section{Conclusions}

One hundred and sixty-two NAC genes from the C. songorica genome were identified, and their basic classification and evolutionary characteristics were analyzed. A genomewide analysis including gene structure, chromosomal location, synteny, expression profiles, coexpression network and GO annotation of the NAC family in C. songorica was first performed. We highlighted the role of CsNAC transcription factors in response to abiotic stress and CL flower development. These results may be helpful to the follow-up research on the role of the CsNAC genes in dimorphic floret development and stress response.

Supplementary Materials: The following are available online at http://www.mdpi.com/2073-4425/11/8/927/s1. Table S1: Characteristics of CsNAC genes in C. songorica. Table S2: Synteny blocks of NAC genes between C. songorica and rice genome. Table S3: Synteny blocks of CsNAC genes within C. songorica genome. Table S4: Expression pattern of CsNAC genes in different organ. Table S5: GO annotation of DEG between CH and CL flowers. Table S6: Gene list and GO annotation of coexpression genes with CL flower development genes. Table S7: The transcriptome data of CsNAC genes in shoot and root under abiotic stress. Table S8: Gene list and GO annotation analysis of coexpression genes with abiotic stress related genes. Table S9: Primer list for gene-specific primers. Figure S1: Overview of 15 conserved motifs identified through MEME analysis. The raw data of the C. songorica whole-genome sequence were submitted to NCBI, number: PRJNA634005; the genome assembly sequence was submitted to the National Genomics Data Center (NGDC), number: PRJCA002752.

Author Contributions: Formal analysis, F.W.; funding acquisition, J.Z.; investigation, X.Z. and Q.Y.; Supervision, J.Z.; writing—original draft, X.Z.; writing—review and editing, F.W.; Q.M. and J.Z. All authors have read and agreed to the published version of the manuscript.

Funding: This research was funded Gansu Provincial Science and Technology Major Projects (19ZD2NA002), the National Natural Science Foundation of China (31572453), the Gansu Provincial Intellectual Property Program (19ZSCQ044) and the 111 Project (B12002).

Conflicts of Interest: The authors declare no conflict of interest.

\section{References}

1. Mittler, R.; Blumwald, E. Genetic engineering for modern agriculture: Challenges and perspectives. Annu. Rev. Plant Biol. 2010, 61, 443-462. [CrossRef]

2. Wang, H.; Miyazaki, S.; Kawai, K.; Deyholos, M.; Galbraith, D.W.; Bohnert, H.J. Temporal progression of gene expression responses to salt shock in maize roots. Plant Mol. Biol. 2003, 52, 873-891. [CrossRef] [PubMed]

3. Luscombe, N.M.; Thornton, J.M. Protein-DNA interactions: Amino acid conservation and the effects of mutations on binding specificity. J. Mol. Biol. 2002, 320, 991-1009. [CrossRef]

4. Riechmann, J.L.; Heard, J.; Martin, G.; Reuber, L.; Jiang, C.-Z.; Keddie, J.; Adam, L.; Pineda, O.; Ratcliffe, O.; Samaha, R. Arabidopsis transcription factors: Genome-wide comparative analysis among eukaryotes. Science 2000, 290, 2105-2110. [CrossRef] [PubMed]

5. Ooka, H.; Satoh, K.; Doi, K.; Nagata, T.; Otomo, Y.; Murakami, K.; Matsubara, K.; Osato, N.; Kawai, J.; Carninci, P.; et al. Comprehensive analysis of NAC family genes in Oryza sativa and Arabidopsis thaliana. DNA Res. 2003, 10, 239-247. [CrossRef]

6. Olsen, A.N.; Ernst, H.A.; Leggio, L.L.; Skriver, K. NAC transcription factors: Structurally distinct, functionally diverse. Trends Plant Sci. 2005, 10, 79-87. [CrossRef]

7. Souer, E.; van Houwelingen, A.; Kloos, D.; Mol, J.; Koes, R. The no apical meristem gene of Petunia is required for pattern formation in embryos and flowers and is expressed at meristem and primordia boundaries. Cell 1996, 85, 159-170. [CrossRef]

8. Swati, P.; Pranav Pankaj, S.; Srivastava, P.S.; Manoj, P. NAC proteins: Regulation and role in stress tolerance. Trends Plant Sci. 2012, 17, 369-381.

9. Seo, P.J.; Kim, S.G.; Park, C.M. Membrane-bound transcription factors in plants. Trends Plant Sci. 2008, 13, 550-556. [CrossRef]

10. Min, X.; Jin, X.; Zhang, Z.; Wei, X.; Ndayambaza, B.; Wang, Y.; Liu, W. Genome-wideidentification of NAC transcription factor family and functional analysis of the abiotic stress-responsive genes in Medicago sativa $\mathrm{L}$. J. Plant Growth Regul. 2020, 39, 324-337. [CrossRef]

11. Sablowsk, R.W.M.; Meyerowitz, E.M. A homolog of NO APICAL MERISTEM is an immediate target of the floral homeotic genes APETALA3/PISTILLATA. Cell 1998, 92, 93-103. [CrossRef] 
12. Ren, T.; Wang, J.; Zhao, M.; Gong, X.; Wang, S.; Wang, G.; Zhou, C. Involvement of NAC transcription factor SiNAC1 in a positive feedback loop via ABA biosynthesis and leaf senescence in Foxtail millet. Planta 2018, 247, 53-68. [CrossRef] [PubMed]

13. Du, M.; Zhai, Q.; Deng, L.; Li, S.; Li, H.; Yan, L.; Huang, Z.; Wang, B.; Jiang, H.; Huang, T. Closely related NAC transcription factors of tomato differentially regulate stomatal closure and reopening during pathogen attack. Plant Cell 2014, 26, 3167-3184. [CrossRef] [PubMed]

14. Kato, H.; Motomura, T.; Komeda, Y.; Saito, T.; Kato, A. Overexpression of the NAC transcription factor family gene ANAC036 results in a dwarf phenotype in Arabidopsis thaliana. J. Plant Physiol. 2010, 167, 571-577. [CrossRef]

15. Kim, Y.S.; Kim, S.G.; Park, J.E.; Park, H.Y.; Lim, M.H.; Chua, N.H.; Park, C.M. A membrane-mound NAC transcription factor regulates cell division in Arabidopsis. Plant Cell 2006, 18, 3132-3144. [CrossRef]

16. Kim, J.H.; Woo, H.R.; Kim, J.; Lim, P.O.; Lee, I.C.; Choi, S.H.; Hwang, D.; Nam, H.G. Trifurcate feed-forward regulation of age-dependent cell death involving miR164 in Arabidopsis. Science 2009, 323, 1053-1057. [CrossRef]

17. Zhong, R.; Lee, C.; Ye, Z. Evolutionary conservation of the transcriptional network regulating secondary cell wall biosynthesis. Trends Plant Sci. 2010, 15, 625-631. [CrossRef]

18. Zhang, L.; Yao, L.; Zhang, N.; Yang, J.; Zhu, X.; Tang, X.; Calderón-Urrea, A.; Si, H. Lateral root development in potato is mediated by stu-mi164 regulation of NAC transcription factor. Front. Plant Sci. 2018, 9, 383. [CrossRef]

19. Kim, S.G.; Lee, A.K.; Yoon, H.K.; Park, C.M. A membrane-bound NAC transcription factor NTL8 regulates gibberellic acid-mediated salt signaling in Arabidopsis seed germination. Plant J. 2008, 55, 77-88. [CrossRef]

20. Zhang, Z.; Dong, J.; Ji, C.; Wu, Y.; Messing, J. NAC-type transcription factors regulate accumulation of starch and protein in maize seeds. Proc. Natl. Acad. Sci. USA 2019, 116, 11223-11228. [CrossRef]

21. Shan, W.; Kuang, J.; Chen, L.; Xie, H.; Peng, H.; Xiao, Y.; Li, X.; Chen, W.; He, Q.; Chen, J. Molecular characterization of banana NAC transcription factors and their interactions with ethylene signalling component EIL during fruit ripening. J. Exp. Bot. 2012, 63, 5171-5187. [CrossRef] [PubMed]

22. Zhao, D.; Derkx, A.; Liu, D.; Buchner, P.; Hawkesford, M. Overexpression of a NAC transcription factor delays leaf senescence and increases grain nitrogen concentration in wheat. Plant Biol. 2015, 17, 904-913. [CrossRef] [PubMed]

23. Yang, S.D.; Seo, P.J.; Yoon, H.K.; Park, C.M. The Arabidopsis NAC transcription factor VNI2 integrates abscisic acid signals into leaf senescence via the COR/RD genes. Plant Cell 2011, 23, 2155-2168. [CrossRef] [PubMed]

24. Block, A.; Toruño, T.Y.; Elowsky, C.G.; Zhang, C.; Steinbrenner, J.; Beynon, J.; Alfano, J.R. The Pseudomonas syringae type III effector HopD1 suppresses effector-triggered immunity, localizes to the endoplasmic reticulum, and targets the Arabidopsis transcription factor NTL9. New Phytol. 2014, 201, 1358-1370. [CrossRef] [PubMed]

25. Hong, Y.; Zhang, H.; Huang, L.; Li, D.; Song, F. Overexpression of a stress-responsive NAC transcription factor gene ONAC022 improves drought and salt tolerance in rice. Front. Plant Sci. 2016, 7, 4. [CrossRef]

26. Yuan, X.; Wang, H.; Cai, J.; Bi, Y.; Li, D.; Song, F. Rice NAC transcription factor ONAC066 functions as a positive regulator of drought and oxidative stress response. BMC Plant Biol. 2019, 19, 278. [CrossRef]

27. Qu, Y.; Duan, M.; Zhang, Z.; Dong, J.; Wang, T. Overexpression of the Medicago falcata NAC transcription factor MfNAC3 enhances cold tolerance in Medicago truncatula. Environ. Exp. Bot. 2016, 129, 67-76. [CrossRef]

28. Fang, Y.; Liao, K.; Du, H.; Xu, Y.; Song, H.; Li, X.; Xiong, L. A stress-responsive NAC transcription factor SNAC3 confers heat and drought tolerance through modulation of reactive oxygen species in rice. J. Exp. Bot. 2015, 66, 6803-6817. [CrossRef]

29. Hao, Y.; Wei, W.; Song, Q.; Chen, H.; Zhang, Y.; Wang, F.; Zou, H.; Lei, G.; Tian, A.; Zhang, W. Soybean NAC transcription factors promote abiotic stress tolerance and lateral root formation in transgenic plants. Plant J. 2011, 68, 302-313. [CrossRef]

30. Wang, B.; Wei, J.; Song, N.; Wang, N.; Zhao, J.; Kang, Z. A novel wheat NAC transcription factor, TaNAC30, negatively regulates resistance of wheat to stripe rust. J. Integr. Plant Biol. 2018, 60, 432-443. [CrossRef]

31. Li, X.; Wang, Y.; Wei, X.; Tai, J.; Jia, C.; Hu, X.; Trethewey, J.A.K. Planting density and irrigation timing affects seed yield sustainability. Agron. J. 2014, 106, 1690-1696. [CrossRef] 
32. Yan, Q.; Wu, F.; Yan, Z.; Li, J.; Ma, T.; Zhang, Y.; Zhao, Y.; Wang, Y.; Zhang, J. Differential co-expression networks of long non-coding RNAs and mRNAs in Cleistogenes songorica under water stress and during recovery. BMC Plant Biol. 2019, 19, 23. [CrossRef] [PubMed]

33. Xu, P.; Wu, F.; Ma, T.; Yan, Q.; Zong, X.; Li, J.; Zhao, Y.; Kanzana, G.; Zhang, J. Analysis of six transcription factor families explores transcript divergence of cleistogamous and chasmogamous flowers in Cleistogenes songorica. DNA Cell Biol. 2020, 39, 273-288. [CrossRef]

34. Zhang, J.; Duan, Z.; Jahufer, Z.; An, S.; Wang, Y. Stress-inducible expression of a Cleistogenes songorica ALDH gene enhanced drought tolerance in transgenic Arabislopsis thaliana. Plant Omics 2014, 7, 438-444.

35. Duan, Z.; Zhang, D.; Zhang, J.; Di, H.; Wu, F.; Hu, X.; Meng, X.; Luo, K.; Zhang, J.; Wang, Y. Co-transforming bar and CSALDH genes enhanced resistance to herbicide and drought and salt stress in transgenic alfalfa (Medicago sativa L.). Front. Plant Sci. 2015, 6, 1115. [CrossRef]

36. Wu, F.; Zhang, D.; Muvunyi, B.P.; Yan, Q.; Zhang, Y.; Yan, Z.; Cao, M.; Wang, Y.; Zhang, J. Analysis of microRNA reveals cleistogamous and chasmogamous floret divergence in dimorphic plant. Sci. Rep. 2018, 8, 6287. [CrossRef]

37. Yan, Q.; Wu, F.; Ma, T.; Zong, X.; Ma, Q.; Li, J.; Zhao, Y.; Wang, Y.; Zhang, J. Comprehensive analysis of bZIP transcription factors uncovers their roles during dimorphic floret differentiation and stress response in Cleistogenes songorica. BMC Genom. 2019, 20, 760. [CrossRef]

38. Muvunyi, B.P.; Yan, Q.; Wu, F.; Min, X.; Yan, Z.; Kanzana, G.; Wang, Y.; Zhang, J. Mining late embryogenesis abundant (LEA) family genes in Cleistogenes songorica, a xerophyte perennial desert plant. Int. J. Mol. Sci. 2018, 19, 3430. [CrossRef]

39. Nuruzzaman, M.; Manimekalai, R.; Sharoni, A.M.; Satoh, K.; Kondoh, H.; Ooka, H.; Kikuchi, S. Genome-wide analysis of NAC transcription factor family in rice. Gene 2010, 465, 30-44. [CrossRef]

40. Nishiyama, R.; Watanabe, Y.; Tran, L.-S.P.; Le, D.T.; Shinozaki, K.; Mochida, K.; Yamaguchi-Shinozaki, K. Genome-wide survey and expression analysis of the plant-specific NAC transcription factor family in soybean during development and dehydration stress. DNA Res. 2011, 18, 263-276.

41. Wang, N.; Zheng, Y.; Xin, H.; Fang, L.; Li, S. Comprehensive analysis of NAC domain transcription factor gene family in Vitis vinifera. Plant Cell Rep. 2013, 32, 61-75. [CrossRef]

42. Zhu, G.; Chen, G.; Zhu, J.; Zhu, Y.; Lu, X.; Li, X.; Hu, Y.; Yan, Y. Molecular characterization and expression profiling of NAC transcription factors in Brachypodium distachyon L. PLoS ONE 2015, 10, e0139794. [CrossRef] [PubMed]

43. Fang, Y.; You, J.; Xie, K.; Xie, W.; Xiong, L. Systematic sequence analysis and identification of tissue-specific or stress-responsive genes of NAC transcription factor family in rice. Mol. Genet. Genom. 2008, 280, 547-563. [CrossRef] [PubMed]

44. Lin, H.; Zhu, W.; Silva, J.C.; Gu, X.; Buell, C.R. Intron gain and loss in segmentally duplicated genes in rice. Genome Biol. 2006, 7, R41. [CrossRef] [PubMed]

45. Hu, R.; Qi, G.; Kong, Y.; Kong, D.; Gao, Q.; Zhou, G. Comprehensive analysis of NAC domain transcription factor gene family in Populus trichocarpa. BMC Plant Biol. 2010, 10, 145. [CrossRef] [PubMed]

46. Hao, Y.; Song, Q.; Chen, H.; Zou, H.; Wei, W.; Kang, X.; Ma, B.; Zhang, W.; Zhang, J.; Chen, S. Plant NAC-type transcription factor proteins contain a NARD domain for repression of transcriptional activation. Planta 2010, 232, 1033-1043. [CrossRef]

47. Liu, M.; Ma, Z.; Sun, W.; Huang, L.; Wu, Q.; Tang, Z.; Bu, T.; Li, C.; Chen, H. Genome-wide analysis of the NAC transcription factor family in tartary buckwheat (Fagopyrum tataricum). BMC Genom. 2019, $20,113$. [CrossRef]

48. Thiel, T.; Graner, A.; Waugh, R.; Grosse, I.; Close, T.J.; Stein, N. Evidence and evolutionary analysis of ancient whole-genome duplication in barley predating the divergence from rice. BMC Evol. Biol. 2009, 9, 209. [CrossRef]

49. Fan, K.; Li, F.; Chen, J.; Li, Z.; Lin, W.; Cai, S.; Liu, J.; Lin, W. Asymmetric evolution and expansion of the NAC transcription factor in polyploidized cotton. Front. Plant Sci. 2018, 9, 47. [CrossRef]

50. Gong, X.; Zhao, L.; Song, X.; Lin, Z.; Gu, B.; Yan, J.; Zhang, S.; Tao, S.; Huang, X. Genome-wide analyses and expression patterns under abiotic stress of NAC transcription factors in white pear (Pyrus bretschneideri). BMC Plant Biol. 2019, 19, 161. [CrossRef]

51. Culley, T.M.; Klooster, M.R. The cleistogamous breeding system: A review of its frequency, evolution, and ecology in angiosperms. Bot. Rev. 2007, 73, 1-30. [CrossRef] 
52. Shibuya, K.; Shimizu, K.; Niki, T.; Ichimura, K. Identification of a NAC transcription factor, EPHEMERAL 1, that controls petal senescence in Japanese morning glory. Plant J. 2014, 79, 1044-1051. [CrossRef] [PubMed]

53. Hendelman, A.; Stav, R.; Zemach, H.; Arazi, T. The tomato NAC transcription factor SINAM2 is involved in flower-boundary morphogenesis. J. Exp. Bot. 2013, 64, 5497-5507. [CrossRef] [PubMed]

54. Langfelder, P.; Horvath, S. WGCNA: An R package for weighted correlation network analysis. BMC Bioinform. 2008, 9, 559. [CrossRef] [PubMed]

55. Hu, H.; Dai, M.; Yao, J.; Xiao, B.; Li, X.; Zhang, Q.; Xiong, L. Overexpressing a NAM, ATAF, and CUC (NAC) transcription factor enhances drought resistance and salt tolerance in rice. Proc. Natl. Acad. Sci. USA 2006, 103, 12987-12992. [CrossRef] [PubMed]

56. Kim, S.G.; Kim, S.Y.; Park, C.M. A membrane-associated NAC transcription factor regulates salt-responsive flowering via FLOWERING LOCUS T in Arabidopsis. Planta 2007, 226, 647-654. [CrossRef]

57. Luo, Y.; Hu, J.; Li, L.; Luo, Y.; Wang, P.; Song, B. Genome-wide analysis of gene expression reveals gene regulatory networks that regulate chasmogamous and cleistogamous flowering in Pseudostellaria heterophylla (Caryophyllaceae). BMC Genom. 2016, 17, 382. [CrossRef]

(C) 2020 by the authors. Licensee MDPI, Basel, Switzerland. This article is an open access article distributed under the terms and conditions of the Creative Commons Attribution (CC BY) license (http://creativecommons.org/licenses/by/4.0/). 